\title{
Adaptación y Análisis Psicométrico de las Escalas de Bienestar Psicológico de Ryff en Adolescentes Argentinos
}

\section{Adaptation and Psychometric Analysis of Ryff's Scales of Psychological Well-Being in Argentinean Adolescents}

\author{
Lisie Karen Meier \\ Universidad Nacional de Formosa \\ Laura Beatriz Oros \\ Consejo Nacional de Investigaciones Científicas y Técnicas
}

\begin{abstract}
El presente estudio tuvo por objetivo adaptar y analizar psicométricamente las Escalas de Bienestar Psicológico de Ryff en una muestra no probabilística de adolescentes argentinos de entre 14 y 16 años. Se utilizó la adaptación al castellano realizada por Díaz et al. (2006) de la versión de 39 ítems propuesta por van Dierendonck (2004). Se realizaron dos estudios: el primero (80 adolescentes y 20 jueces expertos) consistió en el ajuste de 11 ítems y del formato de respuesta de la versión española, según su grado de coherencia teórica, dificultad y claridad lingüística para Argentina, y el segundo (825 adolescentes), en un análisis de las propiedades psicométricas (consistencia interna y validez de constructo) de las Escalas resultantes. El análisis factorial exploratorio mostró la presencia de 4 factores: (a) Autoaceptación, (b) Crecimiento Personal y Propósito en la Vida, (c) Autonomía y (d) Relaciones Positivas con Otras Personas. El análisis factorial confirmatorio mostró un ajuste satisfactorio del modelo, por lo que se concluye que las Escalas adaptadas de 20 ítems son apropiadas para evaluar el bienestar psicológico en la población de adolescentes argentinos.
\end{abstract}

Palabras clave: adaptación, análisis psicométrico, escalas, bienestar, adolescentes

\begin{abstract}
The purpose of this study was to adapt and conduct a psychometric analysis of Ryff's Scales of Psychological WellBeing in a nonprobability sample of Argentinean adolescents aged between 14 and 16 years. The Spanish adaptation by Díaz et al. (2006) of the 39 items version proposed by van Dierendonck (2004) was used. Two studies were conducted: the first (80 adolescents and 20 expert judges) consisted in the adjustment of 11 items and the response format of the Spanish version according to their degree of theoretical consistency, clarity, and linguistic difficulty for Argentina, while the second ( 825 adolescents) was an analysis of the psychometric properties (internal consistency and construct validity) of the resulting Scales. Exploratory factor analysis revealed 4 factors: (a) Self-Acceptance, (b) Personal Growth and Purpose in Life, (c) Autonomy, and (d) Positive Relationships With Others. Confirmatory factor analysis showed a satisfactory level of fit for the model, which makes it possible to conclude that the adapted 20-item Scales are appropriate for assessing the psychological well-being of Argentinean adolescents.
\end{abstract}

Keywords: adaptation, psychometric analysis, scales, well-being, adolescents

Tradicionalmente, la investigación sobre el bienestar se ha realizado a partir de dos concepciones diferentes, aunque relacionadas entre sí: una referida a la felicidad o bienestar hedónico y otra vinculada al desarrollo del potencial humano o bienestar eudaemónico (Deci \& Ryan, 2008). Por un lado, la perspectiva hedónica, con sus raíces ancladas en algunos filósofos griegos, tales como Epicuro (McMahon, 2006), resguarda el placer como el fin supremo de la vida y define su postura del bienestar como una categoría de fenómenos que integran las respuestas emocionales o afectos positivos y negativos de las personas, la satisfacción global con la vida y la satisfacción específica en dominios concretos (Diener, Scollon \& Lucas, 2003; Ryff \& Boylan, 2016), tales como salud, relaciones sociales, ocio e instituciones, entre otros (Vázquez, 2009).

Lisie Karen Meier, Centro Interdisciplinario de Investigaciones en Psicología Matemática y Experimental - Consejo Nacional de Investigaciones Científicas y Técnicas (CONICET), Buenos Aires, Argentina, y Universidad Nacional de Formosa, Argentina; Laura Beatriz Oros, Universidad de la Cuenca del Plata, Corrientes, Argentina, CONICET, Buenos Aires, Argentina, Universidad Adventista del Plata, Libertador San Martín, Argentina, e Instituto Superior Adventista de Misiones, Argentina.

El artículo es parte de la tesis de la primera autora para Optar al Grado de Doctor en Psicología de la Pontificia Universidad Católica Argentina.

La correspondencia relativa a este artículo debe ser dirigida a Lisie K. Meier, Universidad Nacional de Formosa, Maipú 365 (CP 3600), Formosa, Argentina. E-mail: lisiemeier@gmail.com 
Por otro lado, la perspectiva eudaemónica, con sus raíces ancladas en el filósofo griego Aristóteles, entiende el bienestar como un estado de integridad y armonía psicológica. Desde esta tradición, el bienestar se ha centrado en el desarrollo de las virtudes, las capacidades y el crecimiento del ser humano; en otras palabras, se entiende que el bienestar no consiste en maximizar las experiencias positivas y minimizar las negativas (Ryan \& Deci, 2001), sino que se refiere a la vida plena y a la realización de los potenciales humanos (Ryan, Huta \& Deci, 2013).

Algunos autores (Keyes, Shmotkin \& Ryff, 2002) han extendido los límites de la clasificación del bienestar y utilizan el constructo bienestar subjetivo (subjective well-being, SWB) como representante principal de la tradición hedónica y el constructo bienestar psicológico (psychological well-being, PWB) como representante de la tradición eudaemónica.

Los investigadores especializados sostienen que el bienestar subjetivo posee tres componentes: (a) el afecto positivo, (b) el afecto negativo y (c) el componente cognitivo. Algunos de ellos estudian los componentes afectivos y cognitivos de forma separada, ya que consideran que unos son más lábiles y momentáneos en el transcurso del tiempo y otros, más estables y duraderos.

El componente cognitivo es el denominado precisamente como satisfacción vital y resulta de la integración que las personas hacen cuando evalúan cómo les fue en el transcurso de sus vidas o cómo les va en situaciones específicas (Castro Solano, 2009, 2010; Vázquez, 2009; Vázquez, Hervás, Rahona \& Gómez, 2009). Dentro de esta posición teórica, el más utilizado es el modelo tripartito de Diener (1984), que contiene tres componentes: la satisfacción con la vida, el afecto positivo y el afecto negativo.

Existe menos acuerdo entre los investigadores contemporáneos respecto del bienestar psicológico; hasta la fecha, no existe una sola teoría convenida, aunque aparecen típicamente dos puntos de convergencia: un componente de significado y crecimiento personal y la exclusión de un componente afectivo (Disabato, Goodman, Kashdan, Short \& Jarden, 2016; Huta \& Waterman, 2014).

El enfoque del bienestar eudaemónico o bienestar psicológico se ha convertido durante las últimas décadas en un campo floreciente tanto para la práctica clínica como para la investigación científica, especialmente en el marco de la psicología positiva. Ello ha ocurrido posiblemente por el hecho de que sus indicadores fenomenológicos captan detalladamente lo que significa ser humano, es decir, esforzarse, ser proactivo, auténtico, buscar un sentido personal y perseguir el bien supremo para la vida propia. Además, este constructo ha logrado importantes avances, ligándose, mediante objetivos empíricos, a otras variables tales como rasgos de la personalidad, experiencias familiares y ocupacionales, salud física, regulación biológica y procesos neurológicos (Ryff, 2014).

El estudio del bienestar representa, por tanto, un desafío importante para la disciplina psicológica, dado que funciona como un recurso predictor de la salud y la longevidad, de las relaciones sociales y del funcionamiento psicológico óptimo de las personas, constituyendo un indicador útil para evaluar el progreso en psicoterapia y la eficacia de las políticas públicas y de los organismos de gestión en el ámbito de la salud (Castro Solano, 2010; Vázquez \& Hervás, 2008).

Ryff (1989a), quien aparece como uno de los mayores exponentes en el estudio del bienestar psicológico, propone un modelo teórico multidimensional, construido sobre la base de aportaciones clínicas, humanistas, del ciclo vital y de la salud mental, compuesto por seis dimensiones: autoaceptación, relaciones positivas con otras personas, autonomía, dominio del entorno, propósito en la vida y crecimiento personal.

Brevemente, se define (a) la autoaceptación como la actitud positiva hacia el sí mismo, el reconocimiento y la aceptación de lo propio, con un sentimiento positivo hacia lo vivido en el pasado; (b) las relaciones positivas con otras personas como las relaciones cercanas, de confianza, incluyendo la preocupación por el bienestar ajeno y la capacidad de generar empatía, afecto y proximidad con el otro; (c) la autonomía como la independencia, la autodeterminación y la capacidad de autoevaluarse desde los estándares personales; (d) el dominio del entorno como la competencia en el manejo del ambiente y la capacidad de controlar las múltiples actividades externas, haciendo uso efectivo de las oportunidades que brinda el contexto; (e) el propósito en la vida como las metas y el sentido de dirección, junto con el mantenimiento de las creencias que le dan sentido a la vida propia y (f) el crecimiento personal como el sentimiento de desarrollo continuo, de expansión y de crecimiento a través del tiempo (Ryff, 1989b, 2014; Ryff \& Keyes, 1995).

Para medir estas dimensiones teóricas, Ryff (1989b) desarrolló un instrumento conocido como las Escalas de Bienestar Psicológico. La autora generó 80 ítems por dimensión que fueron evaluados sobre la base de su ambigüedad y coherencia con la definición teórica. De ellos, seleccionó 32 ítems por escala, divididos por la 
mitad en ítems positivos y negativos. Los ítems se aplicaron a una muestra piloto de 321 adultos y, tras el estudio de su funcionamiento, se eliminaron 12 ítems por escala, contando cada una finalmente con 20 enunciados. A través del análisis de dicho instrumento, el modelo confirmatorio de seis factores, con uno de segundo orden denominado Bienestar Psicológico, fue el de mejor ajuste, si bien no fue completamente satisfactorio.

Dada la longitud del instrumento, se han propuesto diferentes versiones para la estructura factorial de las Escalas de Bienestar Psicológico. Se cuenta, además, con la versión de 20 ítems por escala de Ryff (1989b), con la versión de Ryff, Lee, Essex y Schumutte (1994) de 14 ítems por escala, con la versión de Ryff y Keyes (1995) de tres ítems por escala con la versión de van Dierendonck (2004) de entre seis y ocho ítems por escala y, por último, con la versión española de Díaz et al. (2006) de entre cuatro y seis ítems.

En los últimos años, las Escalas de Bienestar Psicológico se han replicado en diferentes grupos etarios y a lo largo de numerosos países del mundo, tales como: Japón (Kitamura et al., 2004; Liu, Shono \& Kitamura, 2009), Holanda (van Dierendonck, 2004), Suecia (Lindfors, 2002; Lindfors, Berntsson \& Lundberg, 2006), Reino Unido (Abbott et al., 2006), Francia (Salama-Younes, Ismaïl, Montazeri \& Roncin, 2011), España (Díaz et al., 2006; Freire, Ferradás, Núñez \& Valle, 2017; Tomás Miguel, Meléndez Moral \& Navarro Pardo, 2008), Portugal (Fernandes, Vasconcelos-Raposo \& Teixeira, 2010), México (Loera-Malvaez, Balcázar-Nava, TrejoGonzález, Gurrola-Peña \& Bonilla-Muñoz, 2008; Medina-Calvillo, Gutiérrez-Hernández \& Padrós-Blázquez, 2013), Chile (Chitgian-Urzúa, Urzúa \& Vera-Villarroel, 2013; Gallardo Cuadra \& Moyano-Díaz, 2012; Véliz Burgos, 2012) y Argentina (Aranguren \& Irrazabal, 2015), entre otros.

Los procedimientos más utilizados para aportar datos acerca de las propiedades psicométricas de las Escalas han consistido, en su gran mayoría, en análisis factoriales exploratorios (AFE) y confirmatorios (AFC), para analizar su estructura factorial, y el cálculo de alfa de Cronbach para estimar su consistencia interna.

Díaz et al. (2006) realizaron la adaptación española de la versión holandesa propuesta por van Dierendonck (2004) para las Escalas de Bienestar Psicológico (Ryff, 1989b). Dos traductores bilingües tradujeron la totalidad de los ítems y posteriormente otro traductor bilingüe retrotradujo la versión en castellano de las Escalas y el autor de la versión (van Dierendonck) la comparó con el original. Las diferencias encontradas fueron solventadas mediante la discusión, llegando todos los traductores y el autor a un acuerdo.

La versión resultante de la Escala fue aplicada a una muestra de 467 sujetos, mujeres y varones, de entre 18 y 72 años. La consistencia interna de la versión española de las Escalas de Bienestar Psicológico (Díaz et al., 2006) fue aceptable, obteniéndose en su versión de 39 ítems, divididos en seis dimensiones, un alfa de Cronbach de 0,83 para Autoaceptación, 0,81 para Relaciones Positivas con Otras Personas, 0,73 para Autonomía, 0,71 para Dominio del Entorno, 0,83 para Propósito en la Vida y 0,68 para Crecimiento Personal.

A modo de mejorar los niveles insatisfactorios del AFC de la versión de 39 ítems, Díaz et al. (2006) procedieron al desarrollo de una nueva versión reducida que permitiera un mayor ajuste. La validez factorial de las Escalas de Bienestar Psicológico se comprobó mediante AFC, método de estimación de máxima verosimilitud. El modelo de seis factores con un solo factor de segundo orden, denominado Bienestar Psicológico, presentó los mejores indicadores de ajuste: $\chi^{2}(345, N=467)=615,76, p<0,001$; criterio de información de Akaike $(\mathrm{AIC})=788,16$; índice comparativo de Bentler-Bonett $(\mathrm{CFI})=0,95$; índice no normalizado de Bentler-Bonett $(\mathrm{NNFI})=0,94$; error cuadrático medio residual $(\mathrm{RMR})=0,05$; raíz del error cuadrático medio de aproximación $(\mathrm{RMSEA})=0,04$.

La consistencia interna de la versión resultante de 29 ítems, divididos en seis dimensiones, fue de un alfa de Cronbach de 0,84 para Autoaceptación, 0,78 para Relaciones Positivas con Otras Personas de, 0,70 para Autonomía, 0,82 para Dominio del Entorno, 0,70 para Propósito en la Vida y 0,71 para Crecimiento Personal.

Tal como se ha mencionado anteriormente, Ryff y Keyes (1995) propusieron una estructura factorial conformada por seis dimensiones de primer orden y plantearon la posibilidad de que se incluyera un factor latente de segundo orden, denominado Bienestar Psicológico. Tales resultados fueron apoyados empíricamente por diferentes autores (Díaz, et al., 2006; Kállay \& Rus, 2014; Ryff \& Singer, 2006; van Dierendonck, 2004). Aun así, existen hallazgos que ponen en duda dicha configuración y plantean nuevas alternativas, tales como las de Springer y Hauser (2006), que proponen un factor de segundo orden que comprendería las dimensiones Autoaceptación, Dominio del Entorno, Propósito en la Vida y Crecimiento Personal. Abbott, Ploubidis, Huppert, Kuh y Croudace (2010) respaldan un modelo jerárquico de bienestar psicológico integrado por tres factores: Relaciones Positivas con Otros, Autonomía y un tercer factor de 
segundo orden que nuclea las cuatro dimensiones antes citadas. Kafka y Kozma (2002) proponen una solución de cinco dimensiones de primer orden, al evidenciar que la autoaceptación y el dominio del entorno conforman un único factor. Por último, Tomás, Sancho, Melendez y Mayordomo (2012) obtienen mejores índices de ajuste eliminando las dimensiones Autonomía y Relaciones Positivas con Otros, conformando así una solución de cuatro dimensiones.

Por otro lado, diversos análisis longitudinales han añadido evidencia acerca del carácter distintivo de las dimensiones de las Escalas de Bienestar Psicológico, mostrando perfiles diferenciales de cambio con la edad (Springer, Pudrovska \& Hauser, 2011); consecuentemente, se considera que esta variable podría influir en las características psicométricas del instrumento (Freire et al., 2017).

Loera-Malvaez et al. (2008) realizaron la adaptación de las Escala de Bienestar Psicológico de Ryff en una muestra de adolescentes mexicanos, mujeres y varones, de entre 14 y 18 años de edad, encontrando diferencias con la estructura original propuesta y reportando, mediante un AFE, cuatro factores que denominaron: Autoaceptación, Relaciones Interpersonales, Autonomía y Satisfacción con la Vida. Por otro lado, Medina-Calvillo et al. (2013) encontraron, mediante AFC, que el modelo teórico de seis dimensiones no se ajustaba a los datos en una muestra de adolescentes mexicanos con una media de edad de 19 años.

En Chile, Gallardo Cuadra y Moyano-Díaz (2012) encontraron en una muestra de adolescentes de 14 a 19 años, mediante AFC, que el modelo de seis factores mostraba buen ajuste teórico a los datos, si bien se necesitó de ciertas modificaciones de la Escala a modo de alcanzar los valores considerados adecuados. Los autores, sin embargo, concluyen en que un modelo de cinco factores, agrupando las variables latentes dominio del entorno y propósito en la vida, sería igualmente adecuado, debido a sus resultados satisfactorios.

Por último, Fernandes et al. (2010) también hallaron que el modelo de seis factores mostraba buen ajuste teórico en una muestra de adolescentes portugueses de entre 10 y 20 años, pero, al igual que en la adaptación chilena, requirieron de una serie de modificaciones en las Escalas para lograr los valores adecuados, incluyendo la eliminación de ítems y la covariancia entre residuos. Dichos autores señalan como una gran limitación la falta de una adaptación semántica previa de los ítems a la hora de realizar las comparaciones en el ajuste de los datos, ya que las Escalas originales fueron construidas para población adulta.

Como puede verse, las Escalas de Bienestar Psicológico se han estudiado ampliamente; sin embargo, su construcción original y sus análisis posteriores se han realizado mayormente sobre población joven y adulta y su funcionamiento en el grupo etario adolescente es aún poco estudiado y con resultados muy diversos (ver Fernandes et al., 2010; Gallardo Cuadra \& Moyano-Díaz, 2012; Loera-Malvaez et al., 2008; Medina-Calvillo et al., 2013).

En Argentina existen trabajos que explican y operacionalizan el bienestar psicológico en adolescentes y jóvenes desde la teoría del bienestar psicológico de Ryff, si bien algunos indicadores psicométricos deberían mejorarse y algunas limitaciones ser atendidas en futuras investigaciones. Por ejemplo, Casullo y Castro Solano (2000) realizaron un avance significativo para la evaluación del constructo en población adolescente de entre 13 y 18 años de edad; no obstante, solo contemplaron la medición de cuatro de las Escalas de Ryff, reportaron valores bajos de consistencia interna para la mayoría de ellas y no aportaron información sobre el ajuste a los datos del modelo teórico propuesto.

Por su parte, el estudio de Aranguren e Irrazabal (2015) comprende la evaluación de todas las Escalas de Ryff y, en comparación a los estudios de Casullo y Castro Solano (2000), mejora las propiedades psicométricas, especialmente en lo que se refiere a los valores de consistencia interna, e informa resultados confirmatorios con índices de bondad de ajuste comparativo de Bentler-Bonett (CFI), de Jöreskog (GFI) y normalizado de Bentler-Bonett (NFI) por debajo de los 0,90. Sin embargo, los resultados obtenidos son generalizables a jóvenes y adultos de entre 19 y 35 años de edad, desconociéndose su funcionamiento en muestras de adolescentes.

Tras el análisis de las contribuciones de los trabajos revisados, se considera que la aproximación tanto teórica como empírica orientada al análisis del bienestar psicológico y la creación o adaptación de diferentes instrumentos de medición adecuados continúa siendo una necesidad fundamental (Tomás Miguel et al., 2008), especialmente para mejorar el estudio del funcionamiento positivo y de los elementos que componen una adolescencia saludable.

La teoría y las Escalas de Ryff han demostrado tener una creciente presencia en la ciencia y la práctica en todo el mundo. Es por ello que se consideró relevante evaluar si la versión española de las Escalas de Bienestar Psicológico resultaba válida, confiable y replicable para la población adolescente argentina. 


\section{Método}

\section{Instrumento}

Escalas de Bienestar Psicológico. Se empleó la adaptación al castellano realizada por Díaz et al. (2006) de las Escalas de Bienestar Psicológico (Ryff, 1989b), en base a la versión propuesta por van Dierendonck (2004). Las Escalas evalúan las seis dimensiones propuestas por Ryff (1989a): Autoaceptación (e.g., En general, me siento seguro y positivo conmigo mismo), Relaciones Positivas con Otras Personas (e.g., Siento que mis amistades me aportan muchas cosas), Autonomía (e.g., Tengo confianza en mis opiniones incluso si son contrarias al consenso general), Dominio del Entorno (e.g., Soy bastante bueno manejando muchas de mis responsabilidades en la vida diaria), Propósito en la Vida (e.g., Disfruto haciendo planes para el futuro y trabajar para hacerlos realidad) y Crecimiento Personal (e.g., En general, con el tiempo siento que sigo aprendiendo más sobre mí mismo). El instrumento responde al formato de una escala aditiva tipo Likert compuesta por 39 ítems (seis para cada dimensión de Autoaceptación, Relaciones Positivas con Otras Personas, Dominio del Entorno y Propósito en la Vida, siete para Crecimiento Personal y ocho para Autonomía), a los que los participantes responden utilizando seis alternativas de respuesta: Totalmente en desacuerdo (1), En desacuerdo (2), Parcialmente en desacuerdo (3), Parcialmente de acuerdo (4), De acuerdo (5) y Totalmente de acuerdo (6). Un mayor puntaje revela un mayor bienestar psicológico.

\section{Estudios para la Adaptación y Análisis Psicométrico}

Para la adaptación y análisis psicométrico de las Escalas de Bienestar Psicológico de Ryff en adolescentes argentinos se realizaron dos estudios sucesivos, el Estudio 1 implicó: (a) evaluación de la dificultad y claridad lingüística de los ítems y alternativas de respuesta, por medio de una muestra de jueces adolescentes; (b) modificación y adaptación de los ítems y alternativas de respuesta, en base a las sugerencias de la muestra de jueces adolescentes y (c) evaluación de las modificaciones de los ítems y su correspondencia teórica, por medio de una muestra de jueces expertos.

El Estudio 2 implicó: (a) administración de la prueba a una muestra de adolescentes argentinos, considerando el criterio clásico de 10 sujetos por ítem (Velicer \& Fava, 1998); (b) análisis descriptivo y análisis del poder discriminativo de los ítems, a través de una prueba $t$ para muestras independientes; (c) AFE, mediante el método de máxima verosimilitud y rotación Oblimin directo; (d) AFC, para verificar el ajuste de la estructura al modelo propuesto, por medio del programa AMOS y (e) análisis de la consistencia interna de los ítems, mediante el alfa de Cronbach.

Se obtuvo la autorización y la colaboración de la Dra. Raquel Rodríguez-Carvajal, quien fue parte de la adaptación española y quien brindó toda la información requerida como parte del proceso de adaptación y análisis psicométrico del instrumento en adolescentes argentinos.

A continuación se presentan los dos estudios sucesivos completos con el correspondiente detalle de sus participantes, procedimiento, plan de análisis y resultados obtenidos.

\section{Estudio 1}

El propósito central de este primer estudio fue la adaptación transcultural de las seis Escalas de Bienestar Psicológico de Ryff y la evaluación de su claridad lingüística y coherencia teórica (Meier, 2012).

\section{Método}

Participantes. En primer lugar, se trabajó con una muestra no probabilística incidental de 80 adolescentes argentinos escolarizados. Se seleccionaron 40 hombres y 40 mujeres de entre 14 y 16 años de edad ( $M=15,11, D E=0,79)$ pertenecientes a un colegio semi-privado del nivel medio de educación formal con residencia en la ciudad de Formosa. Se incluyeron los adolescentes, varones y mujeres, que se encontraban asistiendo a la institución educativa y que tenían entre 14 y 16 años de edad. El contacto con la instituciones educativas se realizó mediante el vínculo generado entre los investigadores y los agentes gubernamentales encargados del área educacional, quienes mostraron su interés y destacaron la importancia del tema de la investigación a ejecutar.

En segundo lugar, se trabajó con una muestra no probabilística intencional de 20 profesionales argentinos, de ambos sexos, de entre 27 y 48 años de edad $(M=32,60, D E=4,76)$, investigadores en el área 
de psicología y educación con experiencia en el trabajo con estudiantes universitarios y con residencia en la Ciudad Autónoma de Buenos Aires y en las ciudades de Paraná y Libertador San Martín, Entre Ríos. Fueron contactados personalmente por la primera autora o por correo electrónico, a partir de las sugerencias proporcionadas por miembros del equipo de investigación.

Procedimiento. Al tratarse la primera muestra de menores de edad, se obtuvieron las correspondientes autorizaciones de los directivos encargados de las distintas instituciones educativas donde se seleccionaron los participantes y de los padres, mediante la firma de un consentimiento informado. La administración del cuestionario la realizó la primera autora del presente trabajo, en forma colectiva, en los salones de clases asignados, contando cada uno con un número de entre 25 y 35 adolescentes agrupados por edad. La duración fue de 50 minutos aproximadamente. A cada grupo de alumnos se le explicó el objetivo de la investigación para requerirle su participación voluntaria y se aplicó el cuestionario con una indicación previa sobre las pautas a seguir y la ejemplificación para contestarlo.

Plan de análisis. El primer paso en la adaptación transcultural del instrumento consistió en analizar, a partir de la opinión de los 80 jueces adolescentes, las expresiones utilizadas en la versión española de las Escalas, a modo de conocer si resultaban comprensibles y adecuadas a las expresiones idiomáticas comunes de los adolescentes argentinos. Se les solicitó que sugirieran redacciones alternativas para aquellos ítems que incluían términos poco usuales o de difícil comprensión. También se les señaló que evaluaran la claridad del rango de respuesta preestablecido por los autores. Se realizaron análisis de frecuencias para arribar a los resultados. Las propuestas generadas en esta fase de trabajo fueron cuidadosamente examinadas, dando lugar a una versión argentina preliminar.

El segundo paso consistió en presentar los ítems de ambas versiones (la traducción española y la versión argentina propuesta por las autoras del presente trabajo) a los 20 jueces expertos, quienes debieron compararlos de acuerdo a la coherencia en la expresión lingüística utilizada y a los criterios de adecuación del ítem a la dimensión que pretendía evaluar. Se realizaron análisis de frecuencias y una prueba de $\chi^{2}$ para arribar a los resultados.

\section{Resultados}

A través del análisis de frecuencias se encontró que la adaptación española recomendada por Díaz et al. (2006) incluía expresiones poco usuales para el grupo adolescente argentino, generando dificultades en la compresión de los ítems, por lo que fue necesario realizar una redacción alternativa para 11 de los 39 ítems y para el formato de respuesta de seis puntos.

Los ítems que requirieron modificaciones, sobre la base de que al menos un $10 \%$ de los adolescentes reportara que el ítem era difícil de entender, fueron los siguientes: 5, 10, 12, 15, 18, 21, 22, 27, 35, 36 y 39 (verlos en la Tabla 3). Una vez elaboradas cada una de las redacciones alternativas para estos ítems, fueron presentados a los jueces expertos, junto a las redacciones originales, para que dieran su opinión respecto de la claridad lingüística y conciliación teórica de los nuevos reactivos.

Para el caso de los ítems 5, 15, 18, 22, 27 y 39 y para el formato de respuesta, se presentaron a cada juez dos opciones alternativas por cada ítem para que seleccionaran una de ellas (A o B), ofreciéndose también la posibilidad de que redactaran una tercera opción como sugerencia alternativa. Para algunos ítems los jueces expertos indicaron no solo su preferencia hacia una de las alternativas presentadas, sino también algunas sugerencias de mejora. Estas fueron evaluadas y se incluyeron, según su grado de conveniencia y lógica.

Según los datos de $\chi^{2}$, en prácticamente todos los ítems los jueces mostraron una clara preferencia por una de las dos alternativas presentadas; solo en los ítems 22 y 27 las opiniones de los jueces resultaron divergentes, al igual que respecto del formato de respuestas (ver Tablas 1 y 2). 
Tabla 1

Respuestas de los Jueces Expertos a la Pregunta: ¿Cuál de las Dos Versiones Propuestas Sería Mejor?

\begin{tabular}{lcccccc}
\hline \multirow{2}{*}{ Ítem } & \multicolumn{2}{c}{ A } & \multicolumn{2}{c}{ B } & \multicolumn{2}{c}{ Alternativa } \\
\cline { 2 - 7 } & Frecuencia & $\%$ & Frecuencia & $\%$ & Frecuencia & $\%$ \\
\hline 5 & 12 & 60,0 & 6 & 30,0 & 2 & 10,0 \\
15 & 1 & 5,0 & 15 & 75,0 & 4 & 20,0 \\
18 & 5 & 25,0 & 14 & 70,0 & 1 & 5,0 \\
22 & 7 & 35,0 & 9 & 45,0 & 4 & 20,0 \\
27 & 12 & 60,0 & 8 & 40,0 & - & - \\
39 & 15 & 75,0 & 4 & 20,0 & 1 & 5,0 \\
Formato de respuesta & 11 & 55,0 & 6 & 30,0 & 2 & 10,0 \\
\hline
\end{tabular}

Tabla 2

$\chi^{2}$ en Función a la Elección de los Jueces Expertos en Base a las Alternativas A y B Presentadas a los Ítems y Formato de Respuesta

\begin{tabular}{lrccc}
\hline Ítem & $\chi^{2}$ & $g l$ & $N$ & $p$ \\
\hline 5 & 7,60 & 2 & 20 & 0,022 \\
15 & 16,30 & 2 & 20 & 0,000 \\
18 & 13,30 & 2 & 20 & 0,001 \\
22 & 1,90 & 2 & 20 & 0,387 \\
27 & 0,80 & 1 & 20 & 0,371 \\
39 & 16,30 & 2 & 20 & 0,000 \\
Formato de respuesta & 6,42 & 2 & 20 & 0,400 \\
\hline
\end{tabular}

En el caso del ítem 22, se decidió mantener la opción B, porque era la más clara a criterio de los adolescentes y de las autoras de esta investigación y porque, además, había cierta tendencia de los jueces expertos a preferir esa versión. Con respecto al ítem 27 , se realizó un pequeño ajuste, proponiendo una tercera redacción alternativa para ser puesta a prueba en la muestra piloto. Para el formato de respuestas se optó por la opción de cinco puntos, ya que existía una tendencia importante por parte de los jueces expertos a preferir esa propuesta y a que así lo manifestaban las sugerencias aportadas por la muestra de jueces adolescentes. Además, dicha decisión se consideró pertinente con base en algunos autores que sugieren modificar el formato clásico de respuestas a modo de analizar los diferentes funcionamientos de las Escalas (Springer et al., 2011).

El formato de respuestas incluyó, entonces, las siguientes cinco opciones: (1) Totalmente en desacuerdo, (2) En desacuerdo, (3) Neutro, (4) De acuerdo, (5) Totalmente de acuerdo.

En la Tabla 3 se presentan los 11 ítems originales y modificados tras las adaptaciones realizadas a partir del análisis de los jueces adolescentes y de los jueces expertos.

\section{Estudio 2}

El propósito del siguiente estudio fue analizar la consistencia interna y la validez factorial a nivel exploratorio y confirmatorio de la adaptación argentina de las Escalas de Bienestar Psicológico.

\section{Método}

Participantes. Se trabajó con una muestra no probabilística intencional compuesta por 825 adolescentes del sexo femenino y masculino pertenecientes a cuatro colegios públicos y semi-privados del nivel medio de educación formal con residencia en la ciudad de Formosa. La muestra comprendió 447 mujeres y 378 varones de entre 14 y 16 años de edad $(M=15,14, D E=0,79)$. Se incluyeron los adolescentes, varones y mujeres, que se encontraban asistiendo a las instituciones educativas y que tenían entre 14 y 16 años de edad. 
Tabla 3

Versiones Original y Modificada de los Ítems de las Escalas de Bienestar Psicológico para Adolescentes Argentinos

\begin{tabular}{|c|c|c|}
\hline Ítem & & Versión \\
\hline 5 & $\begin{array}{l}\text { Original } \\
\text { Modificada }\end{array}$ & $\begin{array}{l}\text { Me resulta difícil dirigir mi vida hacia un camino que me satisfaga. } \\
\text { Me resulta difícil guiar mi vida por un camino que me dé satisfacción. }\end{array}$ \\
\hline 10 & $\begin{array}{c}\text { Original } \\
\text { Modificada }\end{array}$ & $\begin{array}{l}\text { Me juzgo por lo que yo creo que es importante, no por los valores que otros piensan que son importantes. } \\
\text { Me juzgo por lo que yo creo que es importante, no por lo que otros piensan que es importante. }\end{array}$ \\
\hline 12 & $\begin{array}{l}\text { Original } \\
\text { Modificada }\end{array}$ & $\begin{array}{l}\text { Soy una persona activa al realizar los proyectos que propuse para mí mismo. } \\
\text { Me esfuerzo por realizar los proyectos que me propuse. }\end{array}$ \\
\hline 15 & $\begin{array}{l}\text { Original } \\
\text { Modificada }\end{array}$ & $\begin{array}{l}\text { Tiendo a estar influenciado por la gente con fuertes convicciones. } \\
\text { Las personas con fuertes convicciones (seguros de sí mismos) me influyen fácilmente. }\end{array}$ \\
\hline 18 & $\begin{array}{l}\text { Original } \\
\text { Modificada }\end{array}$ & $\begin{array}{l}\text { Mis objetivos en la vida han sido más una fuente de satisfacción que de frustración para mí. } \\
\text { Los objetivos de mi vida me han producido más satisfacción que desilusión. }\end{array}$ \\
\hline 21 & $\begin{array}{l}\text { Original } \\
\text { Modificada }\end{array}$ & $\begin{array}{l}\text { Tengo confianza en mis opiniones, incluso si son contrarias al consenso general. } \\
\text { Confío en mi propia opinión, incluso si la mayoría no está de acuerdo. }\end{array}$ \\
\hline 22 & $\begin{array}{l}\text { Original } \\
\text { Modificada }\end{array}$ & $\begin{array}{l}\text { Las demandas de la vida diaria a menudo me deprimen. } \\
\text { Muchas veces las exigencias de la vida diaria me deprimen. }\end{array}$ \\
\hline 27 & $\begin{array}{c}\text { Original } \\
\text { Modificada }\end{array}$ & $\begin{array}{l}\text { Es difícil para mí expresar mis propias opiniones en asuntos polémicos. } \\
\text { Es difícil para mí decir lo que pienso cuando se discuten temas polémicos. }\end{array}$ \\
\hline 35 & Original & $\begin{array}{l}\text { Pienso que es importante tener nuevas experiencias que desafíen lo que uno piensa sobre sí mismo y sobre el } \\
\text { mundo. }\end{array}$ \\
\hline 36 & $\begin{array}{c}\text { Modificada } \\
\text { Original }\end{array}$ & $\begin{array}{l}\text { Es importante tener experiencias que pongan a prueba lo que pienso sobre mí mismo y sobre el mundo. } \\
\text { Cuando pienso en ello, realmente con los años no he mejorado mucho como persona. }\end{array}$ \\
\hline 39 & $\begin{array}{l}\text { Modificada } \\
\text { Original } \\
\text { Modificada }\end{array}$ & $\begin{array}{l}\text { Si lo pienso, creo que con los años no he mejorado mucho como persona. } \\
\text { Si me sintiera infeliz con mi situación de vida daría los pasos más eficaces para cambiarla. } \\
\text { Si me sintiera infeliz con mi vida, haría todo lo posible para cambiarla. }\end{array}$ \\
\hline
\end{tabular}

Procedimiento. Al igual que en el Estudio 1, se tomó previo contacto con las instituciones y se obtuvieron las correspondientes autorizaciones de las autoridades educativas y de los padres de los adolescentes, mediante la firma de un consentimiento informado. La administración del cuestionario la realizó la primera autora del presente trabajo en forma colectiva en los salones de clases asignados, con la misma modalidad que en el caso anterior. La duración fue de 30 minutos aproximadamente.

El estudio fue presentado como una investigación sobre la importancia de diversos rasgos de personalidad, emociones y conductas a la hora de evaluar globalmente la salud. A cada grupo de alumnos se les explicó el objetivo de la investigación para requerirles su colaboración y se les solicitó su participación voluntaria, garantizándoles el anonimato y la confidencialidad de sus respuestas. Se aplicó el cuestionario a cada grupo de adolescentes con una explicación previa sobre las pautas a seguir y la ejemplificación para contestarlo.

Plan de análisis. Al ser la muestra razonablemente grande, se la dividió en dos conjuntos de datos independientes, conformados por una selección al azar de los sujetos. Un conjunto de datos quedó conformado por 451 adolescentes, muestra que se utilizó para el AFE, y el otro, por 374 adolescentes, muestra que se utilizó para el AFC. Todos los análisis restantes, relativos a las características de los ítems y la consistencia interna de las Escalas se llevaron a cabo con la muestra total de 825 sujetos.

Se invirtió la puntuación de los ítems expresados en forma negativa y se realizaron los estadísticos descriptivos de los 39 ítems que componen las Escalas de Bienestar Psicológico para verificar su distribución en la muestra utilizada. Luego, con el criterio de grupos contrastantes, se realizó un análisis del poder discriminativo de los ítems a través de una prueba $t$ de Student para muestras independientes. Esto se realizó para observar si existían diferencias entre los sujetos que puntuaban más alto (cuartil superior) y los que puntuaban más bajo (cuartil inferior) en la variable que se está midiendo (Cohen \& Swerdlik, 2002).

Se realizó un AFE a modo de definir los factores que conformarían las Escalas en Argentina y luego se realizó un AFC a modo de comprobar si las Escalas definidas teóricamente se ajustaban a los datos empíricos. También se realizó un análisis de la consistencia interna de las Escalas resultantes mediante el coeficiente alfa de Cronbach. 
Para el AFE, habiéndose constatado la normalidad multivariada de los datos, se empleó el método de máxima verosimilitud (Costello \& Osborne, 2005) y rotación oblicua, utilizando el criterio Oblimin directo $(\delta$ $=0$ ), bajo el supuesto de que los factores estarían relativamente correlacionados entre sí, en coincidencia con los hallazgos de los autores originales de las Escalas (Ryff, 1989b; Ryff \& Keyes, 1995). Para la extracción de factores se utilizó como criterio el gráfico de sedimentación.

Posteriormente, para evaluar la bondad del ajuste se revisaron los siguientes indicadores: $\chi^{2}$, la razón entre $\chi^{2}$ y el número de grados de libertad $\left(\chi^{2} / g l\right)$, los índices de ajuste parciales de carácter absoluto, GFI y el índice ajustado de bondad de ajuste de Jöreskog (AGFI), así como los índices de ajuste incremental, CFI, el índice de ajuste incremental (IFI) y el índice de Tucker Lewis (TLI), y los índices de ajuste de carácter parsimonioso, RMR y RMSEA.

Con relación al estadístico $\chi^{2}$, y teniendo en cuenta que la sensibilidad de esta medida está ligada al tamaño de la muestra (Schumacker \& Lomax, 2004), se consideró el índice $\chi^{2} / g l$, que permite comprobar de forma más real el nivel de ajuste entre el modelo propuesto y los datos empíricos. Los valores menores que 2 indican un excelente ajuste, mientras que valores hasta 5 se consideran aceptables.

Si bien existen diferentes criterios en cuanto a los índices de bondad de ajuste para definir si el modelo se ajusta o no a los datos, se consideró que para que exista un ajuste aceptable, los valores de GFI y AGFI deberían acercarse o superar el valor de 0,90 (Byrne, 2010). Los índices CFI, IFI y TLI también se consideran adecuados con valores superiores a 0,90 (Schumacker \& Lomax, 2004), mientras que los índices de error RMR y RMSEA deberían ser inferiores a 0,08 (Byrne, 2010; Ruiz, Pardo \& San Martín, 2010). Cabe señalar que ha de tenerse en cuenta siempre la complejidad del modelo teórico, debido a que los indicadores de ajuste disminuyen a medida que aumenta el número de parámetros a estimar (Hu \& Bentler, 1999).

\section{Resultados}

Análisis descriptivo. Se reflejaron valores de asimetría y curtosis inferiores a dos, lo que indicó normalidad univariada de los datos (Bollen \& Long, 1993), exceptuando el ítem 24, que presentó una curtosis superior a dos.

Un número importante de ítems presentó puntuaciones medias superiores a cuatro, aunque en general la desviación típica aseguró una capacidad de discriminación dentro de los rangos aceptables. Entre los ítems con medias altas se encontraron los siguientes: $6,12,14,31,32$ y 39.

Realizada la prueba $t$, se conservaron aquellos ítems que mostraron la mayor capacidad discriminativa $(p<0,001)$, descartando los ítems 16 y 34 .

Análisis factorial exploratorio. Se llevó a cabo con los 37 ítems seleccionados, tras los estudios descriptivos y discriminativos correspondientes. Se calculó previamente la medida de adecuación muestral Kaiser-Meyer-Olkin (KMO; Kaiser, 1974), obteniéndose un valor de 0,846, y el test de esfericidad de Barlett que mostró un $\chi^{2}(666, N=451)=3791,82, p<0,001$, lo que indicó que la matriz era factorizable. El gráfico de sedimentación indicó claramente la presencia de tres factores y un leve descenso escalonado hasta los seis factores, desde donde se mantenía constante la pendiente. Por tal motivo, se extrajeron estos seis factores, respetando la estructura original de las Escalas de Bienestar Psicológico. Los seis factores extraídos en su conjunto explicaron el $32,07 \%$ de la varianza.

En la matriz factorial rotada, recogiendo únicamente los pesos superiores a 0,40, pudo observarse que algunos de los ítems que correspondían a cada factor no condecían a los postulados teóricos para cada dimensión; además, algunos ítems eran explicados por más de un factor, presentando una alta complejidad factorial.

Las dimensiones Crecimiento Personal y Propósito en la Vida presentaron ítems que pesaban en un factor común. A su vez, la dimensión Dominio del Entorno resultó poco clara, por lo que debió ser eliminada, debido a la falta de correspondencia de sus ítems a un único factor.

Como resultado de este primer análisis, se desecharon los elementos que saturaban muy alto en más de un factor o que no poseían correspondencia teórica con el factor en el que pesaban, y se eliminaron los factores residuales. Los ítems descartados fueron los siguientes: 3, 10, 11, 14, 15, 21, 22, 24, 25, 27, 29, 30, 32, 33, 35, 36 y 39. A partir de esta depuración, en un segundo análisis, emergieron cuatro factores que agruparon 20 ítems y explicaron el $34,71 \%$ de la varianza. 
En la Tabla 4 se presenta la matriz factorial rotada. El ítem 18 mostró cierta complejidad factorial, pero, debido a que la diferencia entre las saturaciones simultáneas fue mayor a 0,10, se decidió mantenerlo en el factor de mayor saturación.

Tabla 4

Matriz de Estructura de las Escalas de Bienestar Psicológico: Saturación de los Ítems en Cuatro Factores

Factor e ítem

Componente

$1 \quad 2 \quad 3 \quad 4$

Factor 1: Autoaceptación

31. En su mayor parte, me siento orgulloso de quién soy y la vida que llevo.

1. Cuando repaso la historia de mi vida estoy contento con cómo han resultado las cosas.

17. Me siento bien cuando pienso en lo que he hecho en el pasado y lo que espero hacer en el futuro.

7. En general, me siento seguro y positivo conmigo mismo.

18. Los objetivos de mi vida me han producido más satisfacción que desilusión.

19. Me gusta la mayor parte de los aspectos de mi personalidad.

Factor 2: Crecimiento Personal y Propósito en la Vida

12. Me esfuerzo por realizar los proyectos que me propuse.

6. Disfruto haciendo planes para el futuro y trabajar para hacerlos realidad.

37. Tengo la sensación de que con el tiempo me he desarrollado mucho como persona.

28. Soy bastante bueno manejando muchas de mis responsabilidades en la vida diaria.

23. Tengo clara la dirección y el objetivo de mi vida.

38. Para mí, la vida ha sido un proceso continuo de estudio, cambio y crecimiento.

Factor 3: Relaciones Positivas con Otras Personas

2. A menudo me siento solo porque tengo pocos amigos íntimos con quienes compartir mis preocupaciones.

20. Me parece que la mayor parte de las personas tienen más amigos que yo.

8. No tengo muchas personas que quieran escucharme cuando necesito hablar.

26. No he experimentado muchas relaciones cercanas y de confianza.

Factor 4: Autonomía

4. Me preocupa cómo otra gente evalúa las elecciones que he hecho en mi vida.

9. Tiendo a preocuparme sobre lo que otra gente piensa de mí.

5. Me resulta difícil guiar mi vida por un camino que me dé satisfacción.

13. Si tuviera la oportunidad, hay muchas cosas de mí mismo que cambiaría.

Nota. Los ítems que saturan en cada factor se muestran en negrita.

Como se puede apreciar, algunos de los ítems que componen cada factor no corresponden a los postulados originalmente para cada subescala, conformándose nuevas configuraciones de ítem por factor. El Factor 1 mide Autoaceptación (seis ítems), el Factor 2 mide Crecimiento Personal y Propósito en la Vida (seis ítems), el Factor 3 mide Relaciones Positivas con Otras Personas (cuatro ítems) y el Factor 4 mide Autonomía, (cuatro ítems).

Análisis factorial confirmatorio. El modelo a verificar constaba de 20 ítems distribuidos en cuatro factores, con lo cual se confeccionó una estructura simple con cuatro variables latentes, 20 observables y sin covarianza entre los residuos.

Según Bollen (1989), existe normalidad multivariada si el coeficiente de Mardia es inferior a $p(p+2)$, donde $\mathrm{p}$ es el número de variables observadas. Teniendo en cuenta que en este estudio existían 20 variables observadas y el coeficiente de Mardia fue 75,51, se pudo afirmar que existía normalidad multivariada. A partir de ello, se utilizó el procedimiento de ajuste del modelo de máxima verosimilitud.

Los valores obtenidos a través del AFC mostraron un buen ajuste del modelo a los datos. Si bien la prueba de $\chi^{2}$ resultó significativa, $\left.\chi^{2}(164, N=374)=310,26, p<0,001\right)$, el cociente $\chi^{2} / g l$ presentó un valor adecuado, $\chi^{2} / g l=1,89$. Los demás índices evaluados presentaron valores aceptables: GFI =0,92, AGFI =0,90, 
CFI $=0,91$, IFI $=0,91$ y TLI $=0,90$. Los índices de ajuste de carácter parsimonioso, $R M R=0,07 \mathrm{y}$ RMSEA $=0,04$, presentaron valores dentro de los rangos aceptables.

Observando las correlaciones entre las variables latentes (ver Tabla 5), se evidencia una elevada correlación entre los factores Autoaceptación y Crecimiento Personal y Propósito en la Vida, seguida por los factores Autonomía y Relaciones Positivas con Otras Personas. En función de verificar si el ajuste de las Escalas mejoraba uniendo los factores con alta correlación, se llevó a cabo un AFC con dichas modificaciones, pero los resultados mostraron que el ajuste para el modelo desmejoraba notablemente, por lo cual se decidió mantener el modelo anterior.

Tabla 5

Correlaciones entre Factores de las Escalas de Bienestar Psicológico

\begin{tabular}{lllcr}
\hline Factor & & Factor & $\begin{array}{c}\text { Correlación entre } \\
\text { factores }\end{array}$ & $p$ \\
\hline AUA & $\leftrightarrow$ & RP & 0,367 & $<0,001$ \\
AUA & $\leftrightarrow$ & AUT & 0,323 & $<0,001$ \\
AUA & $\leftrightarrow$ & CP y & 0,797 & $<0,001$ \\
CP y PV & $\leftrightarrow$ & RP & 0,096 & 0,190 \\
CP y PV & $\leftrightarrow$ & AUT & 0,114 & 0,147 \\
AUT & $\leftrightarrow$ & RP & 0,558 & $<0,001$ \\
\hline
\end{tabular}

Nota. AUA = Autoaceptación; CP y PV = Crecimiento Personal y Propósito en la Vida; RP = Relaciones Positivas con Otras Personas; AUT = Autonomía.

A modo de comparar la bondad de ajuste de la nueva configuración de cuatro factores con la del modelo original propuesto por Díaz et al. (2006) en base a la versión propuesta por van Dierendonck (2004), se realizó un AFC con los 39 ítems de las Escalas de Bienestar Psicológico, estableciendo un modelo teórico de seis factores y un factor de segundo orden. Los resultados mostraron que la estructura original no presentaba un ajuste satisfactorio en la muestra de adolescentes argentinos. Se analizó también un segundo modelo teórico de seis factores de primer orden, pero sus resultados continuaron siendo insatisfactorios (ver Tabla 6).

Tabla 6

Análisis Factorial Confirmatorio de las Escalas Originales de 39 Ítems (Díaz et al., 2006) en Una Muestra de Adolescentes Argentinos: Índices de Ajuste

\begin{tabular}{lcccccccccc}
\hline Modelo & $\chi^{2}$ & $p$ & $\chi^{2} / g l$ & GFI & AGFI & CFI & IFI & TLI & RMR & RMSEA \\
\hline $\begin{array}{l}\text { Seis factores, } \\
\text { un factor de } \\
\begin{array}{l}\text { segundo } \\
\text { orden }\end{array}\end{array}$ & 2018,0 & $<0,001$ & 2,85 & 0,72 & 0,69 & 0,56 & 0,56 & 0,54 & 0,13 & 0,07 \\
Seis factores & 1658,5 & $<0,001$ & 2,42 & 0,77 & 0,73 & 0,67 & 0,68 & 0,65 & 0,11 & 0,06 \\
\hline
\end{tabular}

Debido a la conveniencia de comparar el ajuste de los diferentes modelos, se tomaron en cuenta otros índices de bondad de ajuste, especialmente desarrollados para la comparación de modelos, tales como AIC, prefiriéndose, sobre la base de este índice, aquel modelo que presente un valor menor, con mejor ajuste cuanto más cercano a cero; y el índice de validación cruzada esperada (ECVI), que también indica un mejor ajuste del modelo a menor valor (Schermelleh-Engel, Moosbrugger \& Müller, 2003).

El modelo de seis factores con un factor de segundo orden presentó un AIC de 2164,00 y un ECVI de 5,80. El modelo de seis factores de primer orden presentó un AIC de 1844,53 y un ECVI de 4,94. Por último, el 
modelo de cuatro factores propuesto en el presente trabajo presentó un AIC de 402,26 y un ECVI de 1,07, exhibiendo mejores índices de bondad de ajuste en comparación a los modelos previos.

Consistencia interna. Se observa en la Tabla 7 que las cuatro subescalas tienen una consistencia interna dentro de límites aceptables, siendo la de Autonomía la de menor consistencia.

Tabla 7

Consistencia Interna de las Escalas de Bienestar Psicológico en una Muestra de Adolescentes Argentinos

\begin{tabular}{lccc}
\hline \multicolumn{1}{c}{ Subescalas } & $\begin{array}{c}\text { Alfa de } \\
\text { Cronbach }\end{array}$ & $\begin{array}{c}\mathrm{N}^{\circ} \mathrm{de} \\
\text { ítems }\end{array}$ & $\begin{array}{c}\mathrm{N}^{\circ} \mathrm{de} \\
\text { casos }\end{array}$ \\
\hline Autoaceptación & 0,77 & 6 & 803 \\
Crecimiento Personal y Propósito en la Vida & 0,72 & 6 & 807 \\
Relaciones Positivas con Otras Personas & 0,70 & 4 & 813 \\
Autonomía & 0,64 & 4 & 819 \\
\hline
\end{tabular}

\section{Discusión}

Los resultados del proceso de adaptación y análisis psicométrico de las Escalas de Bienestar Psicológico en adolescentes argentinos arrojaron una nueva configuración factorial, en comparación a las Escalas de Bienestar Psicológico propuestas por Díaz et al. (2006) en su adaptación española en base a la versión de van Dierendonck (2004). Dichos hallazgos se suman a los resultados obtenidos en población adolescente mexicana (Loera-Malvaez et al., 2008; Medina-Calvillo et al., 2013), los cuales reportaron una falta de correspondencia con la configuración factorial propuesta por Ryff (1989b).

En la versión adaptada para adolescentes argentinos, las dimensiones Crecimiento Personal y Propósito en la Vida se presentan como un factor único y la dimensión Dominio del Entorno no aparece como un factor relevante. Las Escalas quedaron así compuestas por cuatro factores: Autoaceptación, Crecimiento Personal y Propósito en la Vida, Relaciones Positivas con Otras Personas y Autonomía.

Los resultados obtenidos pueden ser explicados desde los estudios realizados por los mismos autores originales de las Escalas (Ryff \& Keyes, 1995), quienes determinaron que el significado que se le da al bienestar psicológico varía a lo largo de las diferentes etapas de la vida, e, incluso, en otros autores que sostienen que las dimensiones evaluadas por las Escalas de Bienestar Psicológico de Ryff tienen un mejor comportamiento en edades comprendidas entre los 25 y 65 años de edad (Vera-Villarroel, Urzúa, Silva, Pavez \& Celis-Atenas, 2013).

Así, la fusión entre las dos dimensiones que conforman el segundo factor podría indicar que durante la adolescencia los objetivos que se proponen los jóvenes para dar sentido a la vida están estrechamente ligados al empeño que ponen para el desarrollo de las potencialidades y el crecimiento personal, aspectos que podrían ir distanciándose conceptualmente a medida que se avanza hacia la etapa adulta, en la que se ha encontrado que ambos dominios tienden a disminuir (Pudrovska, Springer \& Hauser, 2005, Noviembre; Ryff \& Keyes, 1995; Ryff \& Singer, 2008). Sin embargo, este ha sido un tema poco analizado y sería interesante en futuros estudios indagar con mayor profundidad la razón de estas diferencias, a la luz de la psicología evolutiva.

Por otra parte, algunos autores también han mencionado que las puntuaciones obtenidas en las dimensiones Dominio del Entorno y Autonomía aumentan con la edad (Ryff \& Keyes, 1995; Ryff, Kwan \& Singer, 2001; Ryff \& Singer, 2008) siendo características propias de la adultez tardía y la vejez (Cuadra \& Florenzano, 2003; Ryff \& Singer, 2002). Esto podría explicar la desaparición de la dimensión Dominio del Entorno en esta muestra de adolescentes y la menor consistencia interna de la dimensión Autonomía. Es esperable que en esta etapa del ciclo vital el dominio del entorno, que es definido como la capacidad de manejar el ambiente y de controlar las experiencias externas, esté aún en desarrollo y, por lo tanto, no aparezca como un elemento destacado en la estructura del bienestar. El mismo razonamiento podría aplicarse al caso de la autonomía. Debe tenerse en cuenta que la muestra del estudio incluyó a adolescentes desde los 14 años, cuya independencia, con seguridad, se ha incrementado respecto a su pasada niñez, pero aún no 
asume las características de la independencia propiamente dicha que se experimentará en etapas posteriores (Griffa \& Moreno, 2005; Moreno \& del Barrio, 2005; Papalia, Olds \& Feldman, 2009).

Es importante destacar que las Escalas de Bienestar Psicológico fueron formuladas originalmente en los Estados Unidos (Ryff, 1989b) y que, si bien han sido traducidas a distintos idiomas, su impronta sigue portando elementos que pueden resultar ajenos a la cultura latina. La cultura estadounidense estimula la independencia desde muy temprano, en tanto que la cultura latina tiene una modalidad más protectora, con familias mayoritariamente unidas (affiliatives). "Tener en cuenta estas diferencias culturales no reduce el poder predictivo de la teoría sino que por el contrario incrementa su capacidad de generalización" (Fernández, Pérez, Alderete, Richaud \& Fernández Liporace, 2010, p. 65).

A pesar de que los factores hallados por el presente trabajo no coinciden plenamente con la propuesta de Ryff (1989b) ni con los resultados de van Dierendonck (2004) y Díaz et al. (2006), se consideraron apropiados teóricamente, en tanto que tres de los factores arrojados - Autoaceptación, Relaciones Positivas con Otras Personas y Autonomía - coinciden en modo similar con estudios previos y el nuevo factor, denominado Crecimiento Personal y Propósito de Vida, une dimensiones entre las que se han informado altas correlaciones por otros autores (Kafka \& Kozma, 2002). La eliminación de la dimensión Dominio del Entorno $\mathrm{y}$, a su vez, la falta de coincidencia de algunos de los ítems con respecto a los factores originales, podría verse relacionada, como se ha mencionado con anterioridad, con factores evolutivos y/o culturales relacionados con la expresión y el funcionamiento del bienestar.

En relación al AFC, pudo observarse que los valores obtenidos a través de los índices para evaluar la bondad de ajuste del modelo propuesto de cuatro factores mostraron un buen nivel de ajuste. Los valores de AIC y ECVI muestran un mejor ajuste en el modelo presentado, en comparación con el modelo propuesto por los autores originales. Ha de tenerse en consideración, sin embargo, que dichos modelos presentan cambios sustanciales en el lenguaje utilizado, en la cantidad de ítems y el orden de los factores presentados, lo que dificulta una comparación a niveles estadísticamente significativos pero, no por ello, a niveles funcionalmente significativos (Murray \& Johnson, 2013).

En coincidencia con los autores originales de las Escalas (Ryff \& Keyes, 1995) y con la adaptación chilena de Chitgian-Urzúa et al. (2013) y Véliz Burgos (2012), en la presente adaptación se encontraron altas correlaciones entre algunas de las variables latentes. Springer y Hauser (2006) evaluaron diversos modelos factoriales confirmatorios para las Escalas de Ryff, concluyendo en que la distinción de seis factores era dudosa, dadas sus altas correlaciones. El artículo de Springer y Hauser (2006) posteriormente fue replicado por Ryff y Singer (2006), quienes señalaron que el mejor ajuste global continuaba siendo el de seis factores, lo que no resolvió, sin embargo, el problema de las altas correlaciones entre estos, detalle que nuevamente será expuesto por Springer, Hauser y Freese (2006). Ryff (2014) sostiene actualmente que el problema de las altas correlaciones entre variables latentes posiblemente se deba a la conformación de las dimensiones por un número de ítems cada vez más reducido y no por deficiencias ligadas al modelo teórico, pero dichas suposiciones aún se encuentran bajo análisis.

En relación a la consistencia interna, las subescalas obtuvieron puntuaciones satisfactorias, siendo algo menores a las reportadas en la aplicación española de Díaz et al. (2006) y bastante más altas en comparación al estudio de Casullo (2002) y Casullo y Castro Solano (2000). Resultados similares se encontraron en la adaptación de las Escalas de Bienestar Psicológico en la población adolescente chilena (Gallardo Cuadra \& Moyano-Díaz, 2012), mexicana (Loera-Malvaez et al., 2008) y portuguesa (Fernandes et al., 2010) y en la adaptación para población joven y adulta chilena (Véliz Burgos, 2012; Vera-Villarroel et al., 2013) y argentina (Aranguren \& Irrazabal, 2015).

Cabe mencionar, también, que en la presente adaptación el número de ítems por factor disminuyó tras los análisis, lo cual podría impactar asimismo sobre la consistencia interna obtenida en la versión resultante. Al igual que en la adaptación española de Díaz et al. (2006), pudo observarse que a medida que aumentaba el número de ítems aumentaba también el nivel de consistencia interna de los factores, pero, a su vez, disminuía el ajuste respecto del modelo teórico. Siguiendo, entonces, los lineamientos de Keyes et al. (2002), se mantuvieron los ítems que maximizaran la validez de contenido, priorizando la misma por sobre la consistencia interna.

Teniendo en cuenta las limitaciones del presente trabajo en relación al instrumento analizado, se sugiere que posteriores estudios se dediquen a la formulación de nuevos ítems que quizá se adapten mejor a la expresión y funcionamiento del bienestar en el período adolescente y a la cultura argentina propiamente dicha; probablemente ello actúe a favor de una mejora de la consistencia interna de la dimensión Autonomía, 
logrando mantener, a su vez, la validez de constructo obtenida en la presente adaptación. Sería de gran valor poder ampliar la muestra utilizada a diferentes puntos de residencia en el país, a modo de poder generalizar mejor los resultados a la población adolescente argentina. Se sugiere, por otro lado, ahondar en el análisis vinculado a la modificación de la puntuación de la escala Likert y comparar su funcionamiento respecto a las propuestas utilizadas hasta el momento.

Se propone, además, replicar el AFC en otras muestras a modo de contrastar los resultados obtenidos. Asimismo, teniendo en cuenta que a partir del AFE derivaron importantes modificaciones al instrumento, principalmente referidas a la reducción de ítems y a su nueva agrupación, se vuelve necesario en próximos estudios sumar evidencias de validez de este instrumento, estudiando especialmente la validez convergente con algún otro sistema de medida o la validez nomológica y/o predictiva a partir de hipótesis teóricas previas que sugieran la relación entre los diversos aspectos del bienestar psicológico y otros constructos sociales, afectivos y comportamentales. Esto permitiría garantizar, al menos relativamente, que, a pesar de las modificaciones realizadas, el instrumento continúa evaluando el constructo de bienestar psicológico tal como se pretende.

Finalmente, sería recomendable analizar la estabilidad de estas Escalas a través de un estudio testretest, para aportar más indicadores acerca de su confiabilidad.

\section{Conclusiones}

La versión obtenida en esta investigación fue adaptada rigurosamente y muestra evidencias de consistencia interna. A pesar de mostrar ciertas diferencias con las Escalas de Bienestar Psicológico originales, sigue presentando una estructura coherente con la conceptualización teórica del bienestar psicológico planteada por Ryff, por lo que se considera aceptable su uso en la población adolescente argentina, quedando como desafío el estudio de otros indicadores de validez y confiabilidad que puedan complementar los hallazgos aquí reportados.

\section{Referencias}

Abbott, R. A., Ploubidis, G. B., Huppert, F. A., Kuh, D. \& Croudace, T. J. (2010). An evaluation of the precision of measurement of Ryff's Psychological Well-Being Scales in a population sample. Social Indicators Research, 97, 357-373. https://doi.org/10.1007/s11205009-9506-x

Abbott, R. A., Ploubidis, G. B., Huppert, F. A., Kuh, D., Wadsworth, M. E. J. \& Croudace, T. J. (2006). Psychometric evaluation and predictive validity of Ryff's psychological well-being items in a UK birth cohort sample of woman. Health and Quality of Life Outcomes, 4, Artículo 76. https://doi.org/10.1186/1477-7525-4-76

Aranguren, M. \& Irrazabal, N. (2015). Estudio de las propiedades psicométricas de las Escalas de Bienestar Psicológico de Ryff en una muestra de estudiantes argentinos. Ciencias Psicológicas, 9(1), 73-83. Extraído de http://www.scielo.edu.uy/pdf/cp/v9n1/v9n1a08.pdf

Bollen, K. A. (1989). Structural equations with latent variables. New York, NY: John Wiley \& Sons. https://doi.org/10.1002/9781118619179

Bollen, K. A. \& Long, J. S. (Eds.) (1993). Testing structural equation models. Newbury Park, CA: SAGE.

Byrne, B. M. (2010). Structural equation modeling with AMOS: Basic concepts, applications, and programming (2a ed.). New York, NY: Routledge.

Castro Solano, A. (2009). El bienestar psicológico: cuatro décadas de progreso. Revista Interuniversitaria de Formación del Profesorado, 66, 43-72. Extraído de http://www.redalyc.org/pdf/274/27419066004.pdf

Castro Solano, A. (2010). El estudio del bienestar psicológico. En A. Castro Solano (Comp.), Fundamentos de psicología positiva (pp. 4368). Buenos Aires, Argentina: Paidós.

Casullo, M. M. (2002). Evaluación del bienestar psicológico. En M. M. Casullo (Coord.), Evaluación del bienestar psicológico en Iberoamérica (pp. 11-29). Buenos Aires, Argentina: Paidós.

Casullo, M. M. \& Castro Solano, A. (2000). Evaluación del bienestar psicológico en estudiantes adolescentes argentinos. Revista de Psicología de la Pontificia Universidad Católica de Perú, 18, 35-68. Extraído de http://revistas.pucp.edu.pe/index.php/psicologia/article/view/6840

Chitgian-Urzúa, V., Urzúa, A. \& Vera-Villarroel, P. (2013). Análisis preliminar de las Escalas de Bienestar Psicológico en población chilena. Revista Argentina de Clínica Psicológica, 22, 5-14. Extraído de http://www.redalyc.org/pdf/2819/281930494002.pdf

Cohen, R. J. \& Swerdlik, M. E. (2002). Psychological testing and assessment: An introduction to test and measurement (5 ed.). New York, NY: McGraw Hill.

Costello, A. B. \& Osborne, J. W. (2005). Best practice in exploratory factor analysis: Four recommendations for getting the most from your analysis. Practical Assessment, Research \& Evaluation, 10, Artículo 7. Extraído de http://www.pareonline.net/pdf/v10n7.pdf

Cuadra, L. H. \& Florenzano, U. R. (2003). El bienestar subjetivo: hacia una psicología positiva. Revista de Psicología de la Universidad de Chile, 12(1), 83-96. https://doi.org/10.5354/0719-0581.2003.17380

Deci, E. L. \& Ryan, R. M. (2008). Hedonia, eudaimonia, and well-being: An introduction. Journal of Happiness Studies, 9, 1-11. https://doi.org/10.1007/s10902-006-9018-1

Díaz, D., Rodríguez-Carvajal, R., Blanco, A., Moreno-Jiménez, B., Gallardo, I., Valle, C. \& van Dierendonck, D. (2006). Adaptación española de las Escalas de Bienestar Psicológico de Ryff. Psicothema, 18, 572-577. Extraído de http://www.redalyc.org/pdf/727/72718337.pdf

Diener, E. (1984). Subjective well-being. Psychological Bulletin, 95, 542-575. https://doi.org/10.1037/0033-2909.95.3.542

Diener, E., Scollon, C. N. \& Lucas, R. E. (2003). The evolving concept of subjective well-being: The multifaceted nature of happiness. Advances in Cell Aging and Gerontology, 15, 187-219. https://doi.org/10.1016/S1566-3124(03)15007-9 
Disabato, D. J., Goodman, F. R., Kashdan, T. B., Short, J. L. \& Jarden, A. (2016). Different types of well-being? A cross-cultural examination of hedonic and eudaimonic well-being. Psychological Assessment, 28, 471-482. https://doi.org/10.1037/pas0000209

Fernandes, H. M., Vasconcelos-Raposo, J. \& Teixeira, C. M. (2010). Preliminary analysis of the psychometric properties of Ryff's Scales of Psychological Well-Being in Portuguese adolescents. The Spanish Journal of Psychology, 13, 1032-1043. https://doi.org/10.1017/S1138741600002675

Fernández, A., Pérez, E., Alderete, A. M., Richaud, M. C. \& Fernández Liporace, M. (2010). ¿Construir o adaptar tests psicológicos? Diferentes respuestas a una cuestión controvertida. Revista Evaluar, 10, 60-74. Extraído de https://revistas.unc.edu.ar/index.php/revaluar/article/view/459/428

Freire, C., Ferradás, M. M., Núñez, J. C. \& Valle, A. (2017). Estructura factorial de las Escalas de Bienestar Psicológico de Ryff en estudiantes universitarios. European Journal of Education and Psychology, 10, 1-8. https://doi.org/10.1016/j.ejeps.2016.10.001

Gallardo Cuadra, I. \& Moyano-Díaz, E. (2012). Análisis psicométrico de las Escalas Ryff (versión española) en una muestra de adolescentes chilenos. Universitas Psychologica, 11, 931-939. Extraído de http://www.redalyc.org/pdf/647/64724634021.pdf

Griffa, M. C. \& Moreno, J. E. (2005). Claves para una psicología del desarrollo: adolescencia, adultez, vejez (Volumen II). Buenos Aires, Argentina: Lugar.

Hu, L. \& Bentler, P. M. (1999). Cutoff criteria for fit indexes in covariance structure analysis: Conventional criteria versus new alternatives. Structural Equation Modeling, 6, 1-55. https://doi.org/10.1080/10705519909540118

Huta, V. \& Waterman, A. S. (2014). Eudaimonia and its distinction from hedonia: Developing a classification and terminology for understanding conceptual and operational definitions. Journal of Happiness Studies, 15, 1425-1456. https://doi.org/10.1007/s10902-013-9485-0

Kafka, G. J. \& Kozma, A. (2002). The construct validity of Ryff's Scales of Psychological Well-Being (SPWB) and their relationship to measures of subjective well-being. Social Indicators Research, 57, 171-190. https://doi.org/10.1023/A:1014451725204

Kaiser, H. F. (1974). An index of factorial simplicity. Psychometrika, 39, 31-36. https://doi.org/10.1007/BF02291575

Kállay, É. \& Rus, C. (2014). Psychometric properties of the 44-item version of Ryff's Psychological Well-Being Scale. European Journal of Psychological Assessment, 30, 15-21. https://doi.org/10.1027/1015-5759/a000163

Keyes, C. L. M., Shmotkin, D. \& Ryff, C. D. (2002). Optimizing well-being: The empirical encounter of two traditions. Journal of Personality and Social Psychology, 82, 1007-1022. https://doi.org/10.1037/0022-3514.82.6.1007

Kitamura, T., Kishida, Y., Gatayama, R., Matsuoka, T., Miura, S. \& Yamabe, K. (2004). Ryff's Psychological Well-Being Inventory: Factorial structure and life history correlates among Japanese university students. Psychological Reports, 94, 83-103. https://doi.org/10.2466/pr0.94.1.83-103

Lindfors, P. (2002). Positive health in a group of Swedish white-collar workers. Psychological Reports, 91, 839-845. https://doi.org/10.2466/pr0.2002.91.3.839

Lindfors, P., Berntsson, L. \& Lundberg, U. (2006). Factor structure of Ryff's Psychological Well-Being Scales in Swedish female and male white-collar workers. Personality and Individual Differences, 40, 1213-1222. https://doi.org/10.1016/j.paid.2005.10.016

Liu, Q., Shono, M. \& Kitamura, T. (2009). Psychological well-being, depression, and anxiety in Japanese university students. Depression and Anxiety, 26, E99-E105. https://doi.org/10.1002/da.20455

Loera-Malvaez, N., Balcázar-Nava, P., Trejo-González, L., Gurrola-Peña, G. M. \& Bonilla-Muñoz, M. P. (2008). Adaptación de la Escala de Bienestar Psicológico de Ryff en adolescentes preuniversitarios. Neurología, Neurocirugía y Psiquiatría, 41, 90-97. Extraído de http://www.medigraphic.com/pdfs/revneuneupsi/nnp-2008/nnp083-4b.pdf

Medina-Calvillo, M. A., Gutiérrez-Hernández, C. Y. \& Padrós-Blázquez, F. (2013). Propiedades psicométricas de la Escala de Bienestar Psicológico de Ryff en población mexicana. Revista de Educación y Desarrollo, 27, 25-30. Extraído de http://www.cucs.udg.mx/revistas/edu_desarrollo/anteriores/27/027_Padros.pdf

Meier, L. K. (2012). Adaptación transcultural de las Escalas de Bienestar Psicológico de Ryff en adolescentes. En Facultad de Psicología de la Universidad de Buenos Aires, Memorias del IV Congreso Internacional de Investigación y Práctica Profesional en Psicología, XIX Jornadas de Investigación y VIII Encuentro de Investigadores del MERCOSUR: técnicas y procesos de evaluación psicológica, tomo 2 (pp. 128-129). Buenos Aires, Argentina: Ediciones de la Facultad de Psicología-Universidad de Buenos Aires.

McMahon, D. M. (2006). Happiness: A history. New York, NY: Atlantic Monthly Press.

Moreno, A. \& del Barrio, C. (2005). La experiencia adolescente: a la búsqueda de un lugar en el mundo. Buenos Aires, Argentina: Aique.

Murray, A. L. \& Johnson, W. (2013). The limitations of model fit in comparing the bi-factor versus higher-order models of human cognitive ability structure. Intelligence, 41, 407-422. https://doi.org/10.1016/j.intell.2013.06.004

Papalia, D. E., Olds, S. W. \& Feldman, R. D. (1975/2009). Psicología del desarrollo: de la infancia a la adolescencia (11 ${ }^{\mathrm{a}}$ ed.; S. M. Olivares \& G. E. Padilla, Trads.; Título original: A child's world: Infancy through adolescence). México DF, México: McGraw Hill.

Pudrovska, T., Springer, K. W. \& Hauser, R. M. (2005, Noviembre). Does psychological well-being change with age? Ponencia presentada en la reunión anual de la Gerontological Society of America, Orlando, Florida, Estados Unidos.

Ruiz, M. A., Pardo, A. \& San Martín, R. (2010). Modelos de ecuaciones estructurales. Papeles del Psicólogo, 31, 34-45. Extraído de http://www.redalyc.org/articulo.oa?id=77812441004

Ryan, R. M. \& Deci, E. L. (2001). On happiness and human potentials: A review of research on hedonic and eudaimonic well-being. Annual Review of Psychology, 52, 141-166. https://doi.org/10.1146/annurev.psych.52.1.141

Ryan, R. M., Huta, V. \& Deci, E. L. (2013). Living well: A self-determination theory perspective on eudaimonia. En A. Delle Fave (Ed.), The exploration of happiness: Present and future perspectives (pp. 117-139). Dordrecht, Holanda: Springer. https://doi.org/10.1007/978-94-007-5702-8_7

Ryff, C. D. (1989a). Beyond Ponce de Leon and life satisfaction: New directions in quest of successful ageing. International Journal of Behavioral Development, 12, 35-55. https://doi.org/10.1177/016502548901200102

Ryff, C. D. (1989b). Happiness is everything, or is it? Explorations on the meaning of psychological well-being. Journal of Personality and Social Psychology, 57, 1069-1081. https://doi.org/10.1037/0022-3514.57.6.1069

Ryff, C. D. (2014). Psychological well-being revisited: Advances in the science and practice of eudaimonia. Psychotherapy and Psychosomatics, 83, 10-28. https://doi.org/10.1159/000353263

Ryff, C. D. \& Boylan, J. M. (2016). Linking happiness to health: Comparisons between hedonic and eudaimonic well-being. En L. Bruni \& P. L. Porta (Eds.), Handbook of research methods and applications in happiness and quality of life (pp. 53-70). Cheltenham, Reino Unido: Edward Elgar. https://doi.org/10.4337/9781783471171.00009

Ryff, C. D. \& Keyes, C. L. M. (1995). The structure of psychological well-being revisited. Journal of Personality and Social Psychology, 69, 719-727. https://doi.org/10.1037/0022-3514.69.4.719

Ryff, C. D., Kwan, C. M. L. \& Singer, B. H. (2001). Personality and aging: Flourishing agendas and future challenges. En J. E. Birren \& K. W. Schaie (Eds.), Handbook of the psychology of aging ( $5^{\mathrm{a}}$ ed., pp. 477-499). San Diego, CA: Academic Press.

Ryff, C. D., Lee, Y. H., Essex, M. J. \& Schmutte, P. S. (1994). My children and me: Midlife evaluations of grown children and of self. Psychology and Aging, 9, 195-205. https://doi.org/10.1037/0882-7974.9.2.195 
Ryff, C. D. \& Singer, B. H. (2002). From social structure to biology: Integrative science in pursuit of human health and well-being. En C. R. Snyder \& S. J. Lopez (Eds.), Handbook of positive psychology (pp. 541-554). New York, NY: Oxford University Press.

Ryff, C. D. \& Singer, B. H. (2006). Best news yet on the six-factor model of well-being. Social Science Research, 35, 1103-1119. https://doi.org/10.1016/j.ssresearch.2006.01.002

Ryff, C. D. \& Singer, B. H. (2008). Know thyself and become what you are: A eudaimonic approach to psychological well-being. Journal of Happiness Studies, 9, 13-39. https://doi.org/10.1007/s10902-006-9019-0

Salama-Younes, M., Ismaïl, A., Montazeri, A. \& Roncin, C. (2011). Factor structure of the French Ryff's Psychological Well-Being Scales for active older adults. En I. Brdar (Ed.), The human pursuit of well-being: A cultural approach (pp. 203-212). Dordrecht, Holanda: Springer. https://doi.org/10.1007/978-94-007-1375-8_17

Schermelleh-Engel, K., Moosbrugger, H. \& Müller, H. (2003). Evaluating the fit of structural equation models: Tests of significance and descriptive goodness-of-fit measures. Methods of Psychological Research Online, 8(2), 23-74. Extraído de https://www.dgps.de/fachgruppen/methoden/mpronline/issue20/art2/mpr130_13.pdf

Schumacker, R. E. \& Lomax, R. G. (2004). A beginner's guide to structural equation modeling (2a ed.). Mahwah, NJ: Lawrence Erlbaum.

Springer, K. W. \& Hauser, R. M. (2006). An assessment of the construct validity of Ryff's Scales of Psychological Well-Being: Method, mode and measurement effects. Social Science Research, 35, 1080-1102. https://doi.org/10.1016/j.ssresearch.2005.07.004

Springer, K. W., Hauser, R. M. \& Freese, J. (2006). Bad news indeed for Ryff's six-factor model of well-being. Social Science Research, 35, 1120-1131. https://doi.org/10.1016/j.ssresearch.2006.01.003

Springer, K. W., Pudrovska, T. \& Hauser, R. M. (2011). Does psychological well-being change with age? Longitudinal tests of age variations and further exploration of the multidimensionality of Ryff's model of psychological well-being. Social Science Research, 40, 392-398. https://doi.org/10.1016/j.ssresearch.2010.05.008

Tomás, J. M., Sancho, P., Melendez, J. C. \& Mayordomo, T. (2012). Resilience and coping as predictors of general well-being in the elderly: A structural equation modeling approach. Aging \& Mental Health, 16, 317-326. https://doi.org/10.1080.13607863.2011.615737

Tomás Miguel, J. M., Meléndez Moral, J. C. \& Navarro Pardo, E. (2008). Modelos factoriales confirmatorios de las Escalas de Ryff en una muestra de personas mayores. Psicothema, 20, 304-310. Extraído de http://www.redalyc.org/pdf/727/72720221.pdf

van Dierendonck, D. (2004). The construct validity of Ryff's Scale of Psychological Well-Being and its extension with spiritual well-being. Personality and Individual Differences, 36, 629-643. https://doi.org/10.1016/S0191-8869(03)00122-3

Vázquez, C. (2009). La ciencia del bienestar psicológico. En C. Vázquez \& G. Hervás (Coords.), La ciencia del bienestar: fundamentos de una psicología positiva (pp. 13-46). Madrid, España: Alianza.

Vázquez, C. \& Hervás, G. (2008). Salud positiva: del síntoma al bienestar. En C. Vázquez \& G. Hervás (Eds.), Psicología positiva aplicada (pp. 17-40). Bilbao, España: Desclée de Brouwer.

Vázquez, C., Hervás, G., Rahona, J. J. \& Gómez, D. (2009). Bienestar psicológico y salud: aportaciones desde la psicología positiva. Anuario de Psicología Clínica y de la Salud, 5, 15-28. Extraído de http://institucionales.us.es/apcs/doc/APCS_5_esp_15-28.pdf

Velicer, W. F. \& Fava, J. L. (1998). Effects of variable and subject sampling on factor pattern recovery. Psychological Methods, 3, 231251. https://doi.org/10.1037/1082-989X.3.2.231

Véliz Burgos, A. (2012). Propiedades psicométricas de la Escala de Bienestar Psicológico y su estructura factorial en universitarios chilenos. Psicoperspectivas, 11(2), 143-163. https://doi.org/10.5027/psicoperspectivas-Vol11-Issue2-fulltext-196

Vera-Villarroel, P., Urzúa, A., Silva, J. R., Pavez, P. \& Celis-Atenas, K. (2013). Escala de Bienestar de Ryff: análisis comparativo de los modelos teóricos en distintos grupos de edad. Psicologia: Reflexão e Crítica, 26, 106-112. https://doi.org/10.1590/S010279722013000100012

Fecha de recepción: Febrero de 2017.

Fecha de aceptación: Marzo de 2018. 